\title{
Indien:
}

\section{Alternative Medizin}

\author{
M. Kühni
}

Seit dem 1. Juli 1999 wurden in der Schweiz folgende komplementärmedizinische Behandlungen als Pflichtleistungen für die Krankenkassen aufgenommen: Homöopathie, anthroposophische Medizin, Neuraltherapie, Phytotherapie, chinesische Medizin, Akupunktur.

Die nachfolgenden Bemerkungen stützen sich auf zahlreiche Reisen in Indien und gezielte Interviews mit Patienten und Ärzten.

In Indien sind als komplementär-medizinische Methoden vom Staate anerkannt:

- Ayurvedische Medizin;

- Homöopathie;

- Yunani-(Unani)-Medizin;

- Siddhi-Medizin.

Wie auch bei uns werden von den verschiedenen Ärzten und Helfern noch zahlreiche weitere Methoden der Diagnose und Behandlung durchgeführt, wie z.B. Reiki, chinesische Medizin, Heilen mit ägyptischen Symbolen, Akupressur, Akupunktur, Lichtakupunktur, Bachblüten, Farbtherapie usw. Praktisch jeder Inder konsultiert seinen Astrologen, welcher oft mit Hilfe von Horoskop und Handlesen eine Diagnose seiner Übel stellt und auch medizinische Ratschläge gibt. Zusätzlich gibt es auch viele Heiler im Zusammenhang mit der Religion: Von Gott besessene Menschen geben ihre Kraft durch Berührung, Besprechung oder auf eine andere Weise weiter. Manchmal werden die Kranken auch zum Grabmal eines Heiligen gebracht (Dargah) und dort in Trance geheilt. Im folgenden möchten wir uns auf die staatlich anerkannten Heilverfahren beschränken.

\section{Ayurvedische Medizin}

Die ayurvedische Medizin besteht seit Jahrtausenden. Sie wurde von Weisen, heiligen Männern (Rishis) entwickelt, die ihrerseits die Inspirationen von Gott erhielten. Gesundheit und Krankheit werden durch die drei Prinzipien (Doshas) verursacht und unterhalten: Vata als Prinzip der Bewegung und der Luft, des Äthers, Pitta als dasjenige der Verdauung und des Metabolismus, des Feuers, des Wassers und Kapha, als Prinzip der Stabilität, der Energie, des Wassers und

Korrespondenz:

Dr. med. M. Kühni

Stockacker 24

CH-2564 Bellmund

der Erde. Diese Prinzipien stehen in einem ursprünglichen Gleichgewicht, und im Krankheitsfalle wird dasselbe gestört. Die Diagnose wird durch ein paar physikalische Untersuchungen und durch das anamnestische Gespräch gestellt. Die wichtigste Untersuchung ist die Pulspalpation. Diese braucht eine jahrelange Erfahrung, und es gibt einzelne vollendete Meister, die allein aus der Pulspalpation, selbst ohne Gespräch, die Diagnose stellen können. Andere Untersuchungen sind die Beobachtung der Zunge, der Augen, der Lippen, der Nägel, des Gesichtes. Durch Betrachtung von Urin und Stuhl werden weitere Schlüsse gezogen (blosser Augenschein). Der Patient wird also bei der Untersuchung nicht ausgezogen. In der klassischen Form werden keine Röntgen- oder chemischen Untersuchungen durchgeführt. Allerdings bringen die Patienten zur Konsultation oft die Röntgenbilder oder sonstige Befunde der allopathischen Medizin mit. Die Kunst des Arztes besteht darin, das verlorene Gleichgewicht wieder herzustellen. Die Behandlung besteht aus Diätbesprechung, Instruktion über allgemeine Verhaltensregeln, wie das Meiden von Drogen, Rauchen, Alkohol, regelmässigem und genügendem Schlaf usw. sowie aus der Verabreichung von Medikamenten aus der vegetabilen, animalischen und mineralischen Welt. Je nachdem werden auch sehr teure Medikamente hergestellt, z. B. aus Gold oder Diamanten. Oft werden diese durch den Arzt selber in mühseliger Handarbeit fabriziert. So müssen einzelne Medikamente tagelang von Hand gerührt, andere immer frisch zubereitet werden. Allzu mechanische Herstellungsweisen sind verpönt. Die Manufaktur ist traditionell, wie vor Hunderten von Jahren. Chemische Untersuchungen der Medikamente werden nicht durchgeführt. Die pflanzlichen Medikamente werden nach Aspekt und Geschmack beurteilt, eventuell auch durch Angaben über Jahresund Tageszeit der Gewinnung. Andere Massnahmen sind Reinigungen wie Einläufe, Abführmittel, Aderlass, Provokation von Erbrechen, Schwitzen (eine Art Sauna). Medikamente können auf verschiedenste Weise appliziert werden: per os, über die Nase, tropfenweise auf die Stirne oder auf den Kopf (dabei wird eine Art Haube angebracht, die Medikamente werden einfüllt und entfalten dann ihre Wirkung). Oft werden auch Yogaübungen instruiert und zum täglichen Gebrauch verordnet, Massagen werden regelmässig durchgeführt. Seltener erfolgen psychologische Beratungen oder Ratschläge für eine Pilgerschaft. Die Kunst der ayurvedischen Medizin wurde früher ausschliesslich von Vater zu Sohn weitergegeben. Heute bestehen Universitäten. Nach der Matura folgt ein Lehrgang von etwa vier Jahren, mit anschliessender Praxis von mindestens zwei Jahren. Auffallend ist wenn man ayurvedischen Konsultationen beiwohnt - wie wenig den Patienten erklärt wird. Die komplizierte Diagnose über das Ungleichgewicht der Doshas bildet wohl die Grundlage der Diskussionen unter Ärzten. Dem Laien wird einfach das Medikament in die Hand gedrückt, die Verhaltensweisen instruiert und dann kommt der nächste Patient. 


\section{Homöopathie}

Da die Homöopathie in Europa entwickelt wurde und allgemein bekannt ist, in Kürze nur folgendes: Auch hier besteht eine Theorie von einem begnadeten Menschen (Samuel Hahnemann 1755-1843). Dieselbe wird praktisch für alle Erkrankungen angewandt. Auch diese Theorie stammt also aus Zeiten, wo die Kenntnisse und Möglichkeiten der heutigen Medizin noch relativ wenig entwickelt waren. Vieles beruht auf Tradition und Erfahrungen von Generationen von Ärzten.

\section{Yunani-(Unani)-Medizin}

Die Yunani-Medizin wird auf die Erkenntnisse von Hippokrates (* 460 a.c.) zurückgeführt. Durch die grossen arabischen Ärzte des Mittelalters in Spanien und Kleinasien wurden diese Erkenntnisse weitergegeben und gelangten schliesslich nach Indien und Indonesien. Dort wird diese Wissenschaft weiter praktiziert. Im Verlaufe der Jahrhunderte sind weitere Erkenntnisse dazugekommen. Aber auch hier basiert die Diagnose auf alten Theorien mit den vier Körpersäften Blut, gelbe Galle, schwarze Galle und Schleim. Diese müssen im Gleichgewicht sein und werden im Krankheitsfall gestört. Wesentlich sind dabei unter anderem Klimafaktoren, Jahreszeiten, Temperamente (Sanguiniker, Choleriker, Melancholiker, Phlegmatiker), welche die Erkrankungen verursachen oder unterhalten. Die Diagnose lässt sich vorwiegend aus dem Aspekt (Anamnese, allgemeiner Aspekt, Puls, Augen, Mund mit Zunge) stellen, und somit erübrigen sich andere Untersuchungen. Auch der Urin, der Stuhl, das Sputum und das Blut werden nur aspektmässig beurteilt. In Indien gibt es 18 YunaniColleges. Nach der Matura wird auf universitärer Basis während vier Jahren gelernt. In der Folge kommen noch zusätzlich Praktika in Kliniken dazu. Da diese Medizin über arabische Ärzte nach Indien kam, wird sie auf Urdu - einer Sprache vorwiegend der Mohammedaner - unterrichtet. Sie hat aber nichts mit der Religion zu tun. Sie ist ja viel älter als der Islam. Aus Sprachgründen sind aber etwa 75\% der Ärzte Mohammedaner und nur 25\% Hindu. Entsprechend verteilen sich auch die Patienten. Nach der Diagnosestellung, die dem Patienten meist nicht erklärt wird, erfolgt die Behandlung mit vegetabilen, animalischen und mineralischen Medikamenten. Es gibt über 800 erprobte Medikamente. Dieselben werden auch nach dem Aspekt beurteilt. Eine der Leber ähnliche Pflanzenform wird z.B. für Lebererkrankungen angewandt. Eine gewisse Rolle spielt auch die Organtherapie z.B. mit Testes bei Potenzstörungen oder mit Augen bei Problemen der Sehschärfe. Zeitweise erfolgen auch Therapien mit Handauflegen oder mit Wasserapplikationen. Auch hier werden in den Colleges die modernen Erkenntnisse der Medizin gebraucht und zur Diagnosestellung zugezogen. Sowohl von Yunani-Kollegen als auch ayurvedischen Ärzten wird immer wieder betont, dass die beiden
Systeme absolut verschieden seien. Auch der Medikamentenschatz und die Therapien seien unterschiedlich.

\section{Patientenbefragungen}

Willkürlich, entsprechend zufälligen Begegnungen haben wir 38 Patienten während eines einstündigen Interviews über ihre Ärzte ausgefragt. Selbstverständlich ist die Zahl für statistische Aussagen viel zu klein. Auch können die Angaben von Region zu Region wechseln. Die Gespräche erfolgten in englischer Sprache. Es handelte sich also um Menschen mit einer gewissen Schulbildung aus einer mittleren sozialen Schicht aus der Region Poona und Bombay. Das Ziel der Befragung war, herauszufinden, was für Ärzte hauptsächlich und zuerst von den Patienten zugezogen werden.

\section{Tabelle 1}

\begin{tabular}{ll}
\hline Ayurvedische Medizin & 17 \\
\hline Homöopathie & 16 \\
\hline nur Allopathie & 7 \\
\hline Meditationen & 2 \\
\hline Akupunktur & 1 \\
\hline Glauben & $1(2)$ \\
\hline Hausmittel/Pflanzen & 2 \\
\hline Reiki & 2 \\
\hline «Heilpraktiker» & 3 \\
\hline Shiatsu & 1 \\
\hline Total & $51-52$ \\
\hline
\end{tabular}

Oft brauchen Patienten mehr als einen Arzt. Von 38 Patienten brauchen 13 dauernd 2 Ärzte. Deswegen ergibt die Tabelle eine grössere Zahl konsultierter Ärzte als Anzahl Patienten. Ganz im Vordergrund stehen die Konsultationen bei ayurvedischen und homöopathischen Ärzten. Nur ungefähr $1 / 5$ der Patienten hat alleiniges Vertrauen in die allopathische Medizin. Jeder der Patienten aber sagte aus, dass er bei schweren Erkrankungen immer und sofort einen allopathischen Arzt zuziehen würde. Der Kranke macht also selber eine Triage und bestimmt je nach den Befunden seinen Arzt.

\section{Gespräche mit Ärzten}

Im allgemeinen üben die Ärzte ayurvedischer, homöopathischer oder yunanischer Richtung nur ihre Spezialität aus. Bei der langjährigen Ausbildung ist dies ja verständlich. Einzelne allopathische Ärzte bringen in ihre Tätigkeit auch andere medizinische Auffassungen ein. Das Gespräch mit einem Ärzteehepaar (sie Pädiaterin, er Gynäkologe, beide zur Religionsgemeinschaft der Jain gehörig) ergab: Die 
Frauen werden durch Kurse auf die Geburt vorbereitet, wo auch Yoga und Massage gelehrt wird. Oft wird auch das Gespräch auf die Religion gebracht. Während der Geburt wird dann das Gelernte angewandt und es werden Mantras gesprochen. Ein Mantra ist ein kurzer Meditationsspruch, dem grosse Kräfte innewohnen und der repetitiv ausgesprochen oder gesungen wird. Nach der Geburt werden die Frauen in der Babymassage unterrichtet.

In der ayurvedischen und Yunani-Praxis sind die Konsultationen oft sehr kurz: zwei, drei Worte über die Anamnese, Palpation des Pulses und Betrachtung der Zunge, und schon ist die Diagnose gestellt.

\section{Zusammenfassung, Beurteilung, Applikation für die Schweiz}

1. Die indischen komplementärmedizinischen Praktiken weisen eine jahrhunderte-, ja sogar jahrtausendealte Tradition auf.

2. Die Gründer werden hoch verehrt, sind fast göttlich, eine Autorität, und die Grundprinzipien lassen sich nicht in Frage stellen.

3. Sowohl westliche Medizin als auch die Komplementärmedizinen haben eine eigene Sprache, die nur dem Eingeweihten verständlich ist. Ein Vergleich ist deswegen schwierig oder fast unmöglich. Die Konzeptionen sind gänzlich verschieden.

4. Der grösste Teil der indischen Bevölkerung bevorzugt die alternative Medizin.

5. Bei schweren, besonders bei akuten Erkrankungen wird meist sofort allopathische Medizin einschaltet. Jeder Mensch braucht also zwei Ärzte. Er selber macht jedesmal eine Triage.

6. Da viele Patienten weder die entsprechende Sprache der Mediziner kennen und von der Konzeption oft wenig verstehen, sind die Entscheide der Patienten oft eine Glaubenssache.
7. Da die komplementärmedizinischen Wissenschaften davon ausgehen, die Konstitution des Menschen zu beeinflussen, sind die Behandlungen oft langwierig, eine ganzes Leben lang. Yogaübungen, Diäten usw. müssen immer wieder instruiert werden.

8. Die alternativen Medizinen sind vom Staate anerkannt und werden auf universitärem Niveau gelehrt. Es ist also nicht möglich und unverantwortlich, in kurz dauernden Kursen einige Grundkenntnisse zu erwerben und sich dann als ayurvedischer Therapeut anzuschreiben, wie dies in der Schweiz oft zu beobachten ist.

9. Das Vorgehen des schweizerischen Gesundheitsamtes erscheint diskriminatorisch und nicht sehr objektiv: Es werden nur chinesische Behandlungsmethoden anerkannt (Akupunktur, chinesische Medizin). Ebenso alte und bewährte Methoden aus anderen Kulturkreise, z.B. eben aus Indien, werden nicht berücksichtigt.

10. In Indien kommen auch Patienten, welche komplementärmedizinische Praktiken bevorzugen, im akuten Erkrankungsfall nicht ohne allopathische Behandlung aus. Jeder Patient braucht deshalb parallel mehrere Ärzte, Medikamente und Behandlungen. Auf die Schweiz übertragen, lässt sich vermuten, dass auch hier die Anerkennung der Komplementärmedizin zu Doppelspurigkeiten und dadurch zu einer Verteuerung der Medizin führt. Ausserdem neigen die komplementärmedizinischen Behandlungen oft dazu, sich sehr lange hinzuziehen.

11. Fairerweise sollte jedem Versicherungsnehmer die Wahl gelassen werden, ob er sich a) auf allopathische Behandlungsmethoden beschränken oder b) sich den Luxus mehrerer parallel laufender Behandlungen leisten möchte. Dabei sollte er allerdings die anfallenden Mehrkosten durch entsprechend angepasste Prämien selber übernehmen. 